\title{
Developing a Japanese Version of The Short Form of The Expanded Version of The Posttraumatic Growth Inventory (PTGI-X-SF-J): A Cross-Sectional Study
}

Rei Oshiro ( $\nabla$ re-oshiro@huhs.ac.jp )

Hyogo University of Health Sciences: Hyogo Iryo Daigaku https://orcid.org/0000-0001-5079-8012

Takafumi Soejima

Kobe University: Kobe Daigaku

\section{Sachiko Kita}

National Center for Child Health and Development Research Center: Kokuritsu Kenkyu Kaihatsu Hojin

Kokuritsu Seiiku Iryo Kenkyu Center Kenkyujo

\section{Kayla Benson}

Oakland University

\section{Satoshi Kibi}

The University of Tokyo: Tokyo Daigaku

\section{Koichi Hiraki}

Chinzeigakuin University

\section{Kiyoko Kamibeppu}

The University of Tokyo: Tokyo Daigaku

\section{Kanako Taku}

Oakland University

\section{Research}

Keywords: Posttraumatic growth, Posttraumatic Growth Inventory, PTG assessment, Japanese

Posted Date: July 27th, 2021

DOl: https://doi.org/10.21203/rs.3.rs-716600/v1

License: (9) (1) This work is licensed under a Creative Commons Attribution 4.0 International License. Read Full License 


\section{Abstract \\ Background}

The 10-item short form of the Posttraumatic Growth Inventory (PTGI-SF) has been utilized worldwide. However, there is no Japanese version. Furthermore, the PTGI-SF does not capture a broader category of existential spiritual growth that has been incorporated in the more recent Expanded version of the PTG Inventory (PTGI-X). Thus, we developed a Japanese version of the Short Form (PTGI-X-SF-J), which reflects more diverse perspectives on existential and spiritual growth.

\section{Methods}

A cross-sectional study using questionnaires was conducted in Japan. The first sample consisted of 408 university students, and the second sample comprised 284 university students. Exploratory factor analysis was performed using data from the first sample. Confirmatory factor analysis was conducted, and reliability and validity were confirmed using the second sample.

\section{Results}

The PTGI-X-SF-J is composed of 10 items including two items from each of the five subscales of the PTGI-X, similar to the original version of the PTGI-SF, and this model fits the data well. Items concerning existential/spiritual change in the PTGI-X-SF-J were "I feel more connected with all of existence" and "I have a greater sense of harmony with the world," which were not included in the original PTGI-SF. The score of the PTGI-X-SF-J was significantly correlated with the scores of the Core Beliefs Inventory and the Event Related Rumination Inventory-Deliberate; however, no correlation was observed with the scores of the PTSD Checklist for DSM-5.

\section{Conclusions}

We developed the PTGI-X-SF-J using 10 items to measure PTG among people whose first language is Japanese and confirmed its factor structure, reliability, and validity. The PTGI-X-SF-J is useful for assessing PTG more efficiently and accurately as it considers more diverse spiritual/existential experiences of personal growth and reduces physical and psychological burdens due to its brevity.

\section{Background}

Posttraumatic growth (PTG) refers to the positive psychological changes experienced as a result of the struggle with highly stressful and potentially traumatic life events [1]. Triggering events include not only serious life-threatening events but also any seismic experience that has a life-changing influence [1]. Such events include various adversities such as natural disasters, accidents, illnesses, and relationship 
issues. Thus, PTG can be experienced not only by the person who has directly experienced the event but also by his/her family members and those who witnessed it [2-7]. Previous studies have demonstrated that the degree of PTG is associated with posttraumatic stress disorder (PTSD) symptoms [8, 9], social support [10-12], and coping strategies [13]. In addition, PTG could contribute to psychological distress [14], affect the functional and social dimensions of quality of life (QOL) and well-being [14, 15], and moderate the relationship between PTSD symptoms and QOL [16]. Thus, it is important for healthcare professionals to accurately assess PTG perceived by persons who directly or indirectly experience a potentially traumatic or life-altering event.

The Posttraumatic Growth Inventory (PTGI) is most frequently used to assess PTG worldwide [17]. This scale consists of 21 items with a five-factor structure: personal strength, new possibilities, relating to others, appreciation of life, and spiritual change [17]. Although the PTGI has been commonly used in research and clinical settings, one major concern is that this scale has relatively many items that may lead to physical and psychological burdens among people, especially those who are struggling with adversities, such as receiving aggressive medical treatments due to illness (e.g., cancer) or dealing with life crises (e.g., natural disasters and violence). To address this concern, the short form of the PTGI (PTGISF) was developed by Cann et al. [18]. It consists of 10 items with five subscales similar to the original version. Each subscale has two items selected using factor loadings according to the structure of the original PTGI. The validity and reliability of the PTGI-SF were tested using data on 1,351 adults in the U.S. The PTGI-SF has been translated into multiple languages such as Portuguese and Spanish, but not Japanese $[19,20,21]$. Thus, there is an urgent need to develop a short form of the PTGI for people whose first language is Japanese to reduce their burdens, save time, and assess PTG among them more efficiently. Further, this development would spur more extensive research on PTG in Japan and the provision of clinical care for Japanese people with PTG.

In 2007, the Japanese version of the PTGI (PTGI-J) was developed by Taku et al. [22], and its reliability and validity were confirmed to clarify PTG experiences among Japanese people and compare them with international samples [23-25]. The PTGI-J has a different factor structure than the original PTGI with four instead of five factors: personal strength, new possibilities, relating to others, and spiritual change and appreciation of life (this last factor was created by combining the two original factors: spiritual change and appreciation of life). The four-factor structure of the PTGI-J, being different from the original five-factor structure, may be due to Japanese culture and norms, especially spirituality and religiosity. A previous study reported that Japanese undergraduate students tended not to evaluate the PTGI items, such as having a stronger religious belief, as positive changes [26]. This may be due to the fact that the majority of Japanese people do not believe in monotheism; thus, they may not share religious beliefs, norms, or culture like Western "religious" people might. In addition, many Japanese people may hold negative stereotypes on spirituality or specific religions because of past incidences, such as terrorism and fraud by a religious cult. Another possible explanation may be the tendency of Japanese people to respect their ancestors and traditional rituals more than adhere to specific religious beliefs [26]. 
To resolve these culture-specific issues and capture more diverse perspectives of personal growth, the Posttraumatic Growth Inventory-Expanded (PTGI-X) version was developed in three countries, that is, the U.S., Japan, and Turkey; this version added four items that capture the broader contents of existential and spiritual changes [27]. These additional items of existential and spiritual change are as follows: "I have greater clarity about life's meaning"; "I feel better able to face questions about life and death"; "I feel more connected with all of existence"; and "I have a greater sense of harmony with the world." The validity and reliability of the PTGI-X were confirmed in the three countries, and later in the 10 countries [28], and it was confirmed to be useful for people with no strong religious beliefs like the Japanese [27]. Therefore, when developing a Japanese version of the short form of the PTGI, items concerning spirituality should be considered based on the PTGI-X rather than the original PTGI.

The purpose of this study was to develop a Japanese version of the Short Form of the Expanded version of the Posttraumatic Growth Inventory (PTGI-X-SF-J) and test its validity and reliability. The results of this study could contribute to minimizing burdens among Japanese respondents and assessing their PTG, including existential/spiritual change, more appropriately and efficiently.

\section{Methods}

\section{Study design}

This study included two sample groups: the first and second samples comprised 408 and 284 Japanese university students, respectively. The first study was conducted from April 2016 to June 2017, and the second was conducted from September 2017 to February 2019. This analysis was conducted from February 2021 to April 2021.

\section{Participants}

The first cross-sectional study used questionnaires and was conducted among Japanese students at three universities in the metropolitan area of Japan. After explaining the purpose of the study to the students, those who agreed to participate and signed the consent form were asked to remain in their classroom and complete the questionnaires on their own pace. Of the 423 students who completed the questionnaires, data on 408 Japanese students (152 men, 255 women, and 1 other) were used for this study after excluding the data of 15 participants who answered that they had never experienced any stressful event in the past.

The second cross-sectional study also used questionnaires and was conducted as part of an international joint study on traumatic exposures and PTG [28]. A total of 642 students were recruited and asked to answer the questionnaire, of which 371 students completed the questionnaire. Finally, data on 284 participants including 96 men, 185 women, and 3 "others" were used for this study after excluding 43 cases of missing data and the data of 44 students who answered that they had never experienced any stressful event in the past.

\section{Measures}




\section{Demographics}

Information on age, gender, nationality, religion, and marital status was collected from the two samples.

\section{Posttraumatic growth}

The Japanese version of the PTGI-X was used to assess PTG in both samples. This scale comprises 25 items with a five-factor structure including four new items regarding existential/spiritual changes: personal strength (four items), new possibilities (five items), relating to others (seven items), appreciation of life (three items), and existential/spiritual change (six items). Participants responded to each item using a six-point Likert scale ranging from 0 (I did not experience this change as a result of my crisis) to 5 (I experienced this change to a very great degree as a result of my crisis). Higher scores indicated greater PTG. Cronbach's alpha was .922 for the first sample and .940 for the second sample.

\section{PTSD symptoms}

The PTSD Checklist for DSM-5 (PCL-5 [29, 30]) was used to assess PTSD symptoms in the second sample. This scale includes 20 items with a four-factor structure: intrusions (five items), avoidance (two items), negative alterations in cognitions and mood (seven items), and alterations in arousal and reactivity (six items). The participants evaluated how much they were bothered by each symptom (i.e., item) using a five-point Likert scale ranging from 0 (not at all) to 4 (extremely). Higher scores indicated more PTSD symptoms. Scores $\geq 31$ indicated probable PTSD. Cronbach's alpha was .951 for the second sample.

\section{Stressful events}

In the two studies, 13 or 19 ad hoc questions were used to ask participants whether they had experienced a stressful event (13 or 19 kinds of stressful events, including natural disasters, accidents or injuries, serious illnesses, serious school or academic problems, and family issues) and the degree of their perceived stressfulness at the time it happened.

\section{Challenge to one's assumptive world}

The Japanese version of the Core Beliefs Inventory (CBI-J [31, 32]) was used in the second sample to assess the degree to which one's assumptive world was shaken or challenged by his/her experienced life crisis. This scale has a one-factor structure including nine items. The participants rated the degree to which they seriously re-examined their core beliefs that were disrupted by a specific event that they experienced on a six-point Likert scale ranging from 0 (not at all) to 5 (to a very great degree). Higher scores indicated a greater tendency to challenge one's core beliefs. Cronbach's alpha was .864 for the second sample.

\section{Ruminative process}


The Japanese version of the Event-Related Rumination Inventory (ERRI-J [32, 33]) was used in the second study to assess intrusive and deliberate ruminations following the occurrence of the event that the participants reported. This scale has a two-factor structure and includes 20 items. The first factor includes 10 items that capture intrusive, automatic, and undesired ruminative thoughts (ERRI-Intrusive [ERRI-I]), and the second factor includes the remaining 10 items that capture deliberate and constructive ruminative thoughts (ERRI-Deliberate [ERRI-D]). The participants rated the frequency of each rumination on a five-point Likert scale from 0 (not at all) to 4 (very often). Higher scores indicated more frequent rumination. Cronbach's alpha was .955 for intrusive rumination and .927 for deliberate rumination among the second sample.

\section{Analysis}

First, descriptive statistics were obtained for the demographic variables, stress levels when a stressful event occurred, and the Japanese version of the various scales, such as the PTGI-X, PCL-5, CBI, and ERRI. Next, an exploratory factor analysis (EFA) of the 25 items of the Japanese version of the PTGI-X (PTGI-XJ) was conducted using data from the first study (i.e., 408 Japanese university students) to examine its five-factor structure by selecting two items for each factor according to their factor loadings (i.e., two items with the highest factor loadings). Subsequently, a confirmatory factor analysis (CFA) of the 10 selected items was conducted using data from the second study (i.e., 284 Japanese students) to confirm its factor structure. The goodness of fit of the data was evaluated using the degrees of freedom $\left(\mathrm{x}^{2} / \mathrm{df}\right)$, goodness of fit index $(\mathrm{GFI})$, comparative fit index (CFI), and root mean square error of approximation (RMSEA) [34]. Regarding the internal consistency (i.e., reliability) of the 10 items of the PTGI-X-SF-J, Cronbach's alphas for the total and five subscale scores and the correlation coefficients between them were calculated using data from the second sample. In addition, intraclass correlation coefficients (ICCs) for the total score and subscale scores between the PTGI-X-J and the PTGI-X-SF-J were calculated using data from the second sample. To test the external validity of the PTGI-X-SF-J, the correlation coefficients between the total and subscale scores of the PTGI-X-SF-J and the scores of PCL-5, stress levels at the time it happened, CBI-J, and ERRI-J were calculated. IBM SPSS version $25.0 \mathrm{~J}$ for Windows (SPSS, Chicago, Illinois, USA) and IBM SPSS Amos version 25.0 (SPSS, Chicago, Illinois, USA) were used for statistical analyses.

\section{Ethical considerations}

The study was approved by the appropriate institutional review board. Informed consent was obtained from all participants.

\section{Results}

\section{Participants' demographics}

Regarding the first sample, the mean age of the participants was $22.2(\mathrm{SD}=7.3)$, and $255(62.5 \%)$ were female. A total of 391 participants (95.8\%) described their nationality as Japanese. Regarding the religion 
they were associated with, 279 participants (68.7\%) answered non-religious, 95 participants $(23.4 \%)$ answered Buddhism religion, and 8 participants (2.0\%) answered Christianity. A total of 384 participants $(94.1 \%)$ were single. In contrast, the mean age of participants in the second sample was 21.2 (SD = 3.2), and $185(65.1 \%)$ were female. Of 284, 281 participants (98.9\%) identified their nationality as Japanese. Overall, 282 participants (99.3\%) were single. The participants' characteristics are presented in Table 1. 
Table 1. Participants' characteristics

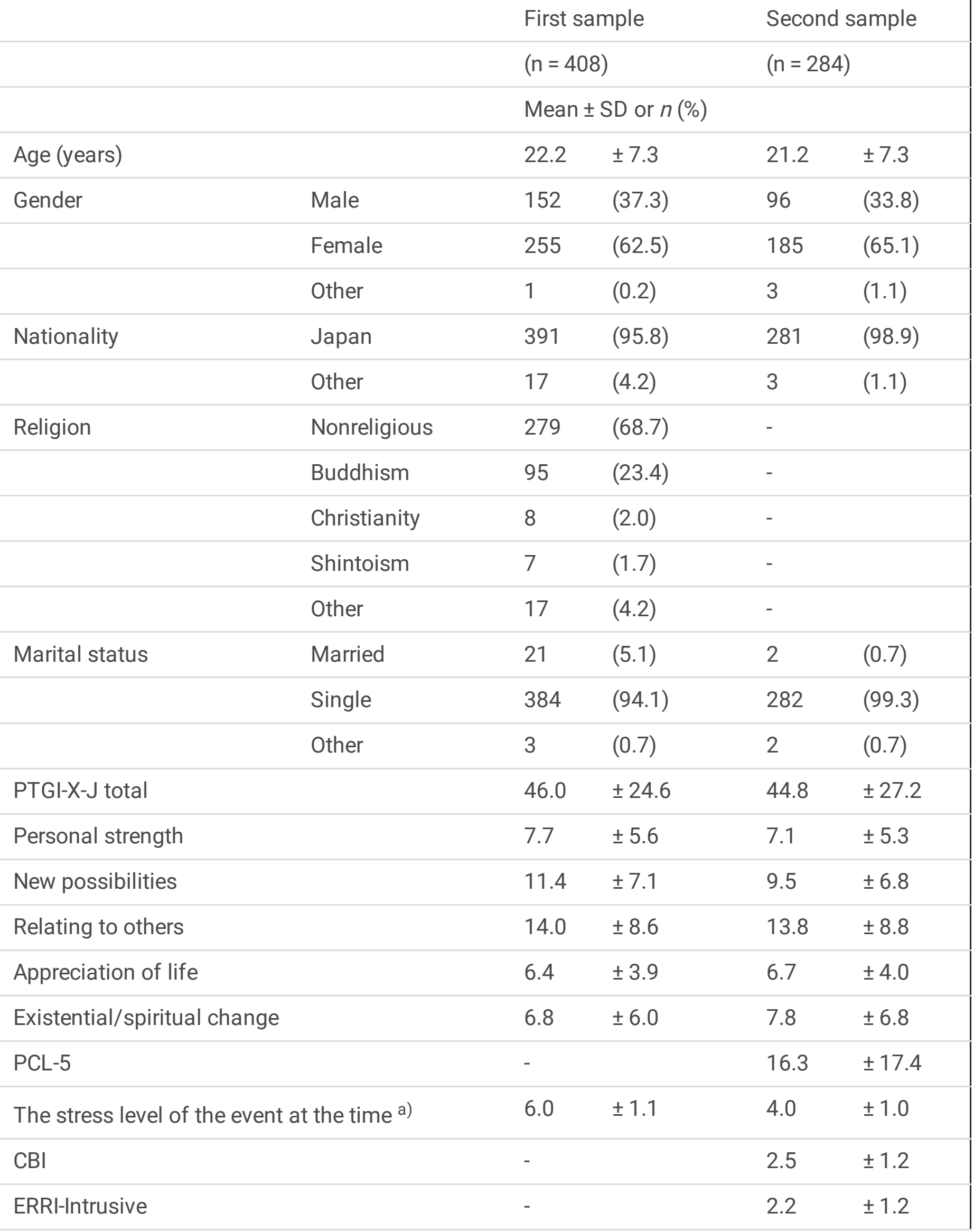




\section{Table 1. Participants' characteristics}

ERRI-Deliberate

1.9

$\pm 1.0$

Notes. Missing values were excluded. Abbreviations. SD = standard deviation.

a) Participants in the first sample responded using a seven-point scale ( $1=$ not at all to $7=$ extremely), and participants in the second sample responded using a five-point scale $(1=$ not at all to $5=$ extremely).

\section{Scores of the scales}

The scores of the scales were also displayed in Table 1. In the first study, the mean scores of the total PTGI-X-J and subscale scores of personal strength, new possibilities, relating to others, appreciation of life, and existential/spiritual change were $46.0(S D=24.6), 7.7(S D=5.6), 11.4(S D=7.1), 14.0(S D=8.6)$, $6.4(S D=3.9)$, and $6.8(S D=6.0)$, respectively. The most common stressful events experienced by participants were serious school/academic problems (27.0\%), romantic relationship problems (13.2\%), and friendship problems (12.5\%). The mean score of participants' perceived stress for a selected event was $6.0(S D=1.1)$.

In the second study, the mean total PTGI-X-J score and subscale scores of personal strength, new possibilities, relating to others, appreciation of life, and existential/spiritual change were $44.8(S D=27.2)$, $7.1(\mathrm{SD}=5.3), 9.5(\mathrm{SD}=6.8), 13.8(\mathrm{SD}=8.8), 6.7(\mathrm{SD}=4.0)$, and $7.8(\mathrm{SD}=6.8)$, respectively. The most common stressful events experienced by participants were interpersonal relationship problems (e.g., bullying, friendships, and relationships with teachers) (19.7\%), natural disasters (12.7\%), and domestic problems (e.g., parental divorce and domestic violence) (12.0\%). The mean score of participants' perceived stress when the event happened was $4.0(S D=1.0)$. The mean score of the total PCL score was 16.3 (SD $=17.4$ ), and 58 participants $(20.4 \%$ ) had probable PTSD. The mean CBI, ERRI-I, and ERRI-D scores were $2.5(S D=1.2), 2.2(S D=1.2)$, and $1.9(S D=1.0)$, respectively.

\section{Exploratory factor analysis}

The results of the EFA using the 25 items of the PTGI-X-J showed a five-factor structure similar to the original version of the PTGI-X (Table 2). Of the 25 items assigned to either factor, the two items with the highest factor loadings in each factor were selected. The factor loadings of the selected items ranged from .491 to .794 . 
Table 2

Items included in the Japanese version of the Expanded version of the Posttraumatic Growth InventoryShort Form and factor loadings from the exploratory factor analysis

\begin{tabular}{|c|c|c|c|c|c|c|}
\hline \multirow[b]{2}{*}{ Items } & & \multicolumn{5}{|c|}{ Factor } \\
\hline & & 1 & 2 & 3 & 4 & 5 \\
\hline $\begin{array}{l}\text { PTGI-X } \\
10\end{array}$ & I know better I can handle difficulties & .794 & .235 & .201 & .153 & .041 \\
\hline $\begin{array}{l}\text { PTGI-X } \\
19\end{array}$ & $\begin{array}{l}\text { I discovered that I'm stronger than I thought I } \\
\text { was }\end{array}$ & .696 & .135 & .090 & .142 & .104 \\
\hline $\begin{array}{l}\text { PTGI-X } \\
14\end{array}$ & $\begin{array}{l}\text { New opportunities are available which wouldn't } \\
\text { have been otherwise }\end{array}$ & .316 & .698 & .093 & -.083 & .115 \\
\hline $\begin{array}{l}\text { PTGI-X } \\
7\end{array}$ & I established a new path for my life & .374 & .686 & .187 & .023 & .179 \\
\hline $\begin{array}{l}\text { PTGI-X } \\
8\end{array}$ & I have a greater sense of closeness with others & .235 & .041 & .753 & .090 & .087 \\
\hline $\begin{array}{l}\text { PTGI-X } \\
6\end{array}$ & $\begin{array}{l}\text { I more clearly see that I can count on people in } \\
\text { times of trouble }\end{array}$ & .182 & .120 & .676 & .083 & .078 \\
\hline $\begin{array}{l}\text { PTGI-X } \\
2\end{array}$ & $\begin{array}{l}\text { I have a greater appreciation for the value of my } \\
\text { own life }\end{array}$ & .093 & -.112 & .162 & .753 & .114 \\
\hline $\begin{array}{l}\text { PTGI-X } \\
13\end{array}$ & I can better appreciate each day & .195 & .291 & .304 & .541 & .104 \\
\hline $\begin{array}{l}\text { PTGI-X } \\
24\end{array}$ & I feel more connected with all of existence & .155 & .245 & .194 & .158 & .639 \\
\hline $\begin{array}{l}\text { PTGI-X } \\
25\end{array}$ & I have a greater sense of harmony with the world & .244 & .299 & .084 & .454 & .491 \\
\hline \multicolumn{7}{|c|}{ Notes. Abbreviation: PTGI-X: Posttraumatic Growth Inventory-Expanded } \\
\hline $\begin{array}{l}\text { The fac } \\
\text { existent }\end{array}$ & $\begin{array}{l}\text { s are 1: personal strength; } 2 \text { : new possibilities; } 3: r \\
\text { /spiritual change. }\end{array}$ & ating & others; & & ation & fe; 5 : \\
\hline
\end{tabular}

\section{Confirmatory factor analysis}

By using the second sample, CFA was performed using 10 items selected by the EFA. The results showed that the model with the five-factor structure using two items for each factor fitted the data well: $\chi 2 / d f=$ $1.64, \mathrm{GFI}=.971, \mathrm{CFI}=.985,=.936$, and RMSEA $=.047$ (Fig. 1). The factor loadings of the 10 selected items ranged from .65 to .86 .

\section{Reliability}

Regarding the internal consistency of the PTGI-X-SF-J using the 10 items, Cronbach's alpha for the total score was .875 . For the subscales, Cronbach's alpha was .678 for personal strength, .807 for new 
possibilities, .707 for relating to others, .753 for appreciation of life, and .671 for existential/spiritual change.

The correlation coefficients between the total score and the subscale scores of the PTGI-X-SF-J ranged from .404 to .794 , indicating moderate to high and positive correlations between them (Table 3).

Table 3

The correlation coefficients between the total and subscale scores

\begin{tabular}{|c|c|c|c|c|c|c|}
\hline & Total & Factor 1 & Factor 2 & Factor 3 & Factor 4 & Factor 5 \\
\hline Total & - & $.740 * \star$ & $.730 \star \star$ & $.794^{\star \star}$ & $.778^{\star \star}$ & $.775^{\star \star}$ \\
\hline Factor 1 & & - & $.533^{\star \star}$ & $.468^{\star \star}$ & $.437 \star \star$ & $.481^{\star \star}$ \\
\hline Factor 2 & & & - & $.433^{\star *}$ & .404 ** & $.469 \star \star$ \\
\hline Factor 3 & & & & - & $.562^{\star \star}$ & $.595^{\star \star}$ \\
\hline Factor 4 & & & & & - & .551 *夫 \\
\hline Factor 5 & & & & & & - \\
\hline
\end{tabular}

The ICC for the total score between the PTGI-X-J and the PTGI-X-SF-J was .821. The ICCs for the subscale scores between the PTGI-X-J and the PTGI-X-SF-J were .863 for personal strength, .825 for new possibilities, .699 for relating to others, .949 for appreciation of life, and .728 for existential/spiritual change.

\section{Validity}

Regarding the external validity of the PTGI-X-SF-J, the variables significantly correlated with the total score of the PTGI-X-SF-J were the scores of the CBI and ERRI-D. The score of the first factor "appreciation of life" was significantly correlated with the scores of the stress levels and ERRI-D. The score of the second factor "new possibilities" was significantly correlated with the scores of CBI, ERRI-I, and ERRI-D. The score of the third factor "personal strength" was significantly correlated with the scores of the CBI and ERRI-D. The score of the fourth factor "relating to others" was significantly correlated with the scores of the CBI, ERRI-I, and ERRI-D. The score of the fifth factor "existential/spiritual change" was significantly correlated with the scores of the stress levels, CBI, and ERRI-D. Details are shown in Table 4. 
Table 4

The correlation coefficients between PTGI-X-SF-J and PCL-5, the stress levels experienced at the time of the event, $\mathrm{CBI}$, and ERRI

\begin{tabular}{|c|c|c|c|c|c|}
\hline PTGI-X-SF-J & PCL-5 & Stress level & CBI & ERRI-I & ERRI-D \\
\hline Total & -.027 & -.109 & $.249 * \star$ & .086 & $.369 * \star$ \\
\hline Factor 1 & -.003 & -.075 & $.200 \star \star$ & .057 & $.340 \star \star$ \\
\hline Factor 2 & -.005 & -.027 & $.372^{\star \star}$ & $.163^{\star \star}$ & $.432^{\star \star}$ \\
\hline Factor 3 & -.016 & .008 & $.124^{\star}$ & $.128 *$ & $.253^{\star \star}$ \\
\hline Factor 4 & -.083 & $-.159 *$ & .081 & -.027 & $.197 \star \star$ \\
\hline Factor 5 & .068 & $-.155^{\star \star}$ & $.251^{\star \star}$ & .036 & $.269 * \star$ \\
\hline \multicolumn{6}{|c|}{$\begin{array}{l}\text { Note. Abbreviations: PTGI-X-SF-J, Japanese version of the Expanded version of the Post Traumatic } \\
\text { Growth Inventory-Short Form; PCL-5, PTSD Checklist for DSM-5; CBI, Core Beliefs Inventory; ERRI-D/I, } \\
\text { Event-Related Rumination Inventory-Deliberate/Intrusive }\end{array}$} \\
\hline \multicolumn{6}{|c|}{$\begin{array}{l}\text { The factors are 1: personal strength; } 2 \text { : new possibilities; } 3 \text { : relating to others; } 4 \text { : appreciation of life; } 5 \\
\text { existential/spiritual change. }{ }^{\star} \mathrm{p}<.05,{ }^{\star \star} \mathrm{p}<.01 \text {. }\end{array}$} \\
\hline
\end{tabular}

\section{Discussion}

This study tested the factor structure, validity, and reliability of the PTGI-X-SF-J using 10 items. The results demonstrated that the PTGI-X-SF-J had a five-factor structure similar to that of the PTGI-X and the original version of the PTGI-SF, and its model showed a good fit to the data. In addition, this study confirmed the moderate to good validity and reliability of the PTGI-X-SF-J using the scores of the stress levels, CBI, and ERRI and Cronbach's alphas.

Although the factor structure of the PTGI-X-SF-J was the same as that of the original version of the PTGISF, the items constructed for the factors of the PTGI-X-SF-J, especially items concerning existential/spiritual change, were different from those of the original version of the PTGI-SF. That is, items for the "existential/spiritual change" factor in the PTGI-X-SF-J were "I feel more connected with all of existence" and "I have a greater sense of harmony with the world," while items concerning "spiritual change" factor in the original PTGI-SF were "I have a strong religious faith" and "I have a better understanding of spiritual matters." The newly selected two items of the PTGI-X-SF-J were the same items evaluated as highly endorsed by Japanese participants in a previous study [25]. The current results reflect more diverse perspectives and better capture the broader domain of existential and spiritual personal growth, instead of narrowly focusing on religiosity. The majority of Japanese people do not believe in a specific religion or in God. Indeed, $68.7 \%$ of the participants in the first study (408 Japanese university students) answered that they did not believe in a specific religion. In addition, a previous study showed that only $30 \%$ of the Japanese population have a specific religious faith, and this percentage is the lowest among developed countries [35]. Although the majority of Japanese people do not believe in a 
specific religion or a higher power, they are likely to have a unique sense of spirituality and traditional spiritual behaviors in daily life [36]. For example, there are traditional spiritual events called "Omiyamair" and "Shichigosan" for which parents take their children to a local shrine to pray for their children's good health and development when the children are one month or three, five, and seven years old because they believe a local god or "Kami" protects them and their family. Other examples include an annual event called "Hatsumoude" for which Japanese people go to a local shrine or any shrine depending on what they want to pray for on the first few days of a new year and a local custom called "Obon" for which Japanese people pray and welcome their ancestors during the four days of August because they believe their ancestors return to this world. Although Japanese people are not likely to strongly believe in a specific region, they believe in different kinds of gods and spiritual existence, including local and other gods living in a shrine, ancestors, animal gods or spirits, and gods or spirits existing in a mountain, river, flowers, and sea; further, they believe in living together and being protected by the above-mentioned gods and spirits [36]. This suggests that when Japanese people experience psychological struggle due to a stressful life event that they experienced and its impact on their lives, they may feel reconnected with "Kami" or gods and spiritual existence around them through exploring the meaning of their struggle for life and development and praying to many "Kamis" or gods or any kind of spiritual existence they believe in or just feel. These culturally unique spiritual experiences were; however, not well captured by the original items such as "having a stronger religious faith" but reasonably well captured by the items we selected based on the PTGI-X-J in the current samples, such as "more connected with all of existence."

Regarding the validity of the PTGI-X-SF-J, the correlations between the total and subscale scores of the PTGI-X-SF-J and the scores of the CBI and ERRI-D were consistent with the results of previous studies. Previous studies indicated that persons with higher PTG are more likely to reconsider what they used to believe in $[32,37,38,39]$ and think deeply, deliberately, and constructively about the meaning of their stressful events $[32,38,39]$. Only two subscales of the PTGI-X-SF-J were shown to be correlated with the ERRI-I. Taku and her colleagues [32] have indicated that during a certain period after a stressful event, people are more likely to experience higher levels of intrusive ruminations (i.e., thinking unconsciously and repeatedly about stressful events) than deliberate ruminations, and this would influence their PTG. Due to the cross-sectional design of this study and the characteristics of the participants (e.g., university students with relatively younger age), the range of duration after a stressful event might be wider than that captured in the present study. Therefore, a clear correlation between the PTGI-X-SF-J and ERRI-I was not reported in this study.

In addition, there was no correlation between the PTGI-X-SF-J and PCL-5. The results did not support the findings of a previous study among Japanese undergraduate students [22] but were overall consistent with studies showing negligible to small positive correlations. Butler et al. (2005) and Zebrack et al. (2015) suggested that the relationship between trauma symptomatology and PTG is not linear and that it has an inverted U-shaped relationship [40,41]. This means that fewer PTSD symptoms are positively correlated with PTG, and more PTSD symptoms are negatively correlated with PTG. The scores of the PCL-5 in this study varied widely, which might have directly led to no linear correlations between the 
variables in this study. Thus, this result should be cautiously interpreted, and further studies are necessary.

\section{Clinical implications}

Developing the PTGI-X-SF-J using only 10 items could contribute to assessing PTG among Japanese people more efficiently by minimizing their burdens, as this would decrease the total time spent to complete the questionnaire. The PTGI-X-SF-J should be more useful when assessing PTG among people with severe physical or psychological distress due to serious, stressful events, such as illnesses (e.g., cancer), accidents, natural disasters, and other life-threatening events to prevent their further burdens when responding to scales. In addition, the PTGI-X-SF-J was developed using items that were more suitable for the broader spirituality of Japanese people. This will lead to a more accurate assessment and understanding of PTG among the Japanese and provide an effective intervention that respects their spirituality and existential experiences, thereby fostering their PTG. Finally, because the PTGI-X-SF-J was developed using the same factor structure (five factors) and the same number of items (i.e., 10 items) as the original version of the PTGI-SF developed in the U.S., this scale can be compared with scales in previous and future studies in other countries. Moreover, it will contribute to understanding the impact of culture on PTG and its mechanism and developing universal and culture-specific interventions to foster PTG experiences.

\section{Limitations}

This study has some limitations. First, the participants of this study were not representative of the general Japanese population; the participants were young university students, most of whom were healthy. Thus, the results of this study should be cautiously interpreted, and a future study should be conducted among a Japanese population with diverse age ranges, socioeconomic backgrounds, and health status to confirm if the PTGI-X-SF-J is applicable to the general Japanese population and to clarify associations between the variables tested in this study. Second, this study used data from two cross-sectional surveys; thus, the test-retest reliability of the PTGI-X-SF-J could not be evaluated. To obtain evidence for the availability of the PTGI-X-SF-J, a future longitudinal study is needed.

\section{Conclusions}

We developed a Japanese version of the PTGI-X-SF (PTGI-X-SF-J) using only 10 items to measure PTG among Japanese people and confirmed its factor structure, reliability, and validity. The results of this study suggest that the PTGI-X-SF-J developed herein should be useful for assessing PTG more efficiently and accurately as it considers the unique spiritual beliefs and norms of Japanese people and reduces their physical and psychological burdens. In addition, the PTGI-SF-X-J should contribute to not only assessing PTG in Japan but also comparing PTG between countries and developing effective and specific interventions that are sensitive to different cultures to improve PTG in the future. 


\section{List Of Abbreviations}

$\mathrm{CBI}$

Core Beliefs Inventory

CFA

Confirmatory factor analysis

CFI

Comparative fit index

EFA

Exploratory factor analysis

ERRI

Event-Related Rumination Inventory

ERRI-D

ERRI-Deliberate

ERRI-I

ERRI-Intrusive

GFI

Goodness of fit index

PCL-5

PTSD Checklist for DSM-5

PTG

Posttraumatic growth

PTGI

Posttraumatic Growth Inventory

PTGI-J

Japanese version of the PTGI

PTGI-SF

Posttraumatic Growth Inventory-Short Form

PTGI-X

Posttraumatic Growth Inventory-Expanded

PTGI-X-J

Japanese version of the PTGI-X

PTGI-X-SF-J

Japanese version of the Short Form of the Expanded version of the Posttraumatic Growth Inventory PTSD

Posttraumatic stress disorder

QOL

Quality of life

RMSEA

Root mean square error of approximation. 


\section{Declarations}

\section{Ethics approval and consent to participate}

The study was approved by the institutional review boards of the two universities, one in Japan and one in the US.

\section{Consent for publication}

Not applicable.

\section{Availability of data and materials}

The datasets used and/or analyzed during the current study are available from the corresponding author upon reasonable request.

\section{Competing interests}

The authors declare that they have no competing interests.

\section{Funding}

This work was supported by a Grant-in-Aid for Early-Career Scientist awarded to Rei Oshiro (grant number 21K17382) and Grant-in-Aid for Early-Career Scientist (B) awarded to Takafumi Soejima (grant number 16K20786).

\section{Authors' contributions}

R.O. and K.T. contributed to the conception and design of this study; R.O., T.S., K.B., S.K., K.H., K.K., and K.T. conducted data collection; R.O. performed the statistical analysis. R.O. and S.K. drafted the manuscript; T.S., K.B., S.K., K.H., K.K., and K.T. critically reviewed the manuscript and supervised the entire study process. All authors read and approved the final manuscript.

\section{Acknowledgements}

We sincerely thank the study participants.

\section{References}

1. Calhoun LG, Tedeschi RG, editors. Facilitating posttraumatic growth: A clinician's guide. Routledge; 1999.

2. Hefferon K, Grealy M, Mutrie N. Post-traumatic growth and life threatening physical illness: A systematic review of the qualitative literature. Br J Health Psychol. 2009;14(2):343-78. 
3. Meyerson DA, Grant KE, Carter JS, Kilmer RP. Posttraumatic growth among children and adolescents: A systematic review. Clin Psychol Rev. 2011;31(6):949-64.

4. Michael C, Cooper M. Post-traumatic growth following bereavement: A systematic review of the literature. Couns Psychol Rev. 2013;28(4):18-33.

5. Picoraro JA, Womer JW, Kazak AE, Feudtner C. Posttraumatic growth in parents and pediatric patients. J Palliat Med. 2014;17(2):209-18.

6. Ulloa E, Guzman ML, Salazar M, Cala C. Posttraumatic growth and sexual violence: A literature review. J Aggress Maltreat Trauma. 2016;25(3):286-304.

7. Casellas-Grau A, Vives J, Font A, Ochoa C. Positive psychological functioning in breast cancer: An integrative review. Breast. 2016;27:136-68.

8. Wu Z, Xu J, Sui Y. Posttraumatic stress disorder and posttraumatic growth coexistence and the risk factors in Wenchuan earthquake survivors. Psychiatry Res. 2016;237:49-54. http://dx.doi.org/10.1016/j.psychres.

9. Liu AN, Wang LL, Li HP, Gong J, Liu XH. Correlation between posttraumatic growth and posttraumatic stress disorder symptoms based on Pearson correlation coefficient: A meta-analysis. J Nerv Ment Dis. 2017;205(5):380-9.

10. Jia X, Ying L, Zhou X, Wu X, Lin C. The effects of extraversion, social support on the posttraumatic stress disorder and posttraumatic growth of adolescent survivors of the Wenchuan earthquake. PLOS ONE. 2015;10:e0121480. http://dx.doi.org/10.1371/journal.pone.0121480.

11. Yu Y, Peng L, Chen L, Long L, He W, Li M, et al. Resilience and social support promote posttraumatic growth of women with infertility: The mediating role of positive coping. Psychiatry Res. 2014;215:401-5. http://dx.doi.org/10.1016/j.psychres.2013.10.032.

12. Zhou $X$, Wu $X$, Zhen $R$. Understanding the relationship between social support and posttraumatic stress disorder/posttraumatic growth among adolescents after Ya'an earthquake: The role of emotion regulation. Psychol Trauma. 2017;9(2):214-21.

13. Zhai J, Newton J, Copnell B. Posttraumatic growth experiences and its contextual factors in women with breast cancer: An integrative review. Health Care Women Int. 2019;40(5):554-80.

14. Husson O, Zebrack B, Block R, Embry L, Aguilar C, Hayes-Lattin B, et al. Posttraumatic growth and well-being among adolescents and young adults (AYAs) with cancer: A longitudinal study. Support Care Cancer. 2017;25(9):2881-90.

15. Gangeri L, Scrignaro M, Bianchi E, Borreani C, Bhoorie S, Mazzaferro V. A longitudinal investigation of posttraumatic growth and quality of life in liver transplant recipients. Prog Transplant. 2018;28(3):236-43.

16. Martz E, Livneh H, Southwick SM, Pietrzak RH. Posttraumatic growth moderates the effect of posttraumatic stress on quality of life in US military veterans with life-threatening illness or injury. $J$ Psychosom Res. 2018;109:1-8.

17. Tedeschi RG, Calhoun LG. The posttraumatic growth inventory: Measuring the positive legacy of trauma. J Trauma Stress. 1996;9(3):455-71. 
18. Cann A, Calhoun LG, Tedeschi RG, Taku K, Vishnevsky T, Triplett KN, et al. A short form of the Posttraumatic Growth Inventory. Anxiety Stress Coping. 2010;23(2):127-37.

19. Lamela D, Figueiredo B, Bastos A, Martins H. Psychometric properties of the Portuguese version of the posttraumatic growth inventory short form among divorced adults. Eur J Psychol Assess. 2014;30(1):3.

20. García FE, Wlodarczyk A. Psychometric properties of the posttraumatic growth inventory-short form among Chilean adults. J Loss Trauma. 2016;21(4):303-14.

21. Castro MC, Delgado JB, Alvarado ER, Rovira DP. Spanish adaptation and validation of the posttraumatic growth inventory-short form. Violence Vict. 2015;30(5):756-69.

22. Taku K, Calhoun LG, Tedeschi RG, Gil-Rivas V, Kilmer RP, Cann A. Examining posttraumatic growth among Japanese university students. Anxiety Stress Coping. 2007;20(4):353-67.

23. Taku K. Commonly defined and individually defined posttraumatic growth in the US and Japan. Pers Individ Dif. 2011;51(2):188-93.

24. Shigemoto Y, Poyrazli S. Factors related to posttraumatic growth in US and Japanese college students. Psychol Trauma Theor Res Pract Policy. 2013;5(2):128-34.

25. Yoshida H, Kobayashi N, Honda N, Matsuoka H, Yamaguchi T, Homma H, et al. Post-traumatic growth of children affected by the great east japan earthquake and their attitudes to memorial services and media coverage. Psychiatry Clin Neurosci. 2016;70(5):193-01.

26. Oshiro R, Kopitz J, Soejima T, Kibi S, Kamibeppu K, Sakamoto S, et al. Perceptions of positive and negative changes for posttraumatic growth and depreciation: Judgments from Japanese undergraduates. Pers Individ Dif. 2019;137:17-21.

27. Tedeschi RG, Cann A, Taku K, Senol-Durak E, Calhoun LG. The posttraumatic growth inventory: A revision integrating existential and spiritual change. J Trauma Stress. 2017;30(1):11-8.

28. Taku K, Tedeschi RG, Shakespeare-Finch J, Krosch D, David G, Kehl D, et al. Posttraumatic growth (PTG) and posttraumatic depreciation (PTD) across ten countries: Global validation of the PTG-PTD theoretical model. Pers Individ Dif. 2021;169:110222.

29. Weathers FW, Litz BT, Keane TM, Palmieri PA, Marx BP, Schnurr PP. The PTSD checklist for DSM-5 (PCL-5). Scale available from the National Center for PTSD. 2013. Retrieved from: http://www.ptsd.va.go.

30. Ito M, Takebayashi Y, Suzuki Y, Horikoshi M. Posttraumatic stress disorder checklist for DSM-5: Psychometric properties in a Japanese population. J Affect Disord. 2019;247:11-9.

31. Cann A, Calhoun LG, Tedeschi RG, Kilmer RP, Gil-Rivas V, Vishnevsky T, et al. The Core Beliefs Inventory: A brief measure of disruption in the assumptive world. Anxiety Stress Coping. 2010;23(1):19-34.

32. Taku K, Cann A, Tedeschi RG, Calhoun LG. Core beliefs shaken by an earthquake correlate with posttraumatic growth. Psychol Trauma. 2015;7(6):563-9. 
33. Cann A, Calhoun LG, Tedeschi RG, Triplett KN, Vishnevsky T, Lindstrom CM. Assessing posttraumatic cognitive processes: The event related rumination inventory. Anxiety Stress Coping. 2011;24(2):13756.

34. Schermelleh-Engel $\mathrm{K}$, Moosbrugger $\mathrm{H}$, Müller $\mathrm{H}$. Evaluating the fit of structural equation models: Tests of significance and descriptive goodness-of-fit measures. Methods Psychol Res. 2003;8(2):2374.

35. Hayashi F, Nikaido K. Religious faith and religious feelings in Japan: Analyses of cross-cultural and longitudinal surveys. Behaviormetrika. 2009;36(2):167-80.

36. Manabe K. The structure of Japanese religiosity [Japanese]. Kwansei gakuin univ sch. sociol J. 2008;104:45-70.

37. Danhauer SC, Russell GB, Tedeschi RG, Jesse MT, Vishnevsky T, Daley K, et al. A longitudinal investigation of posttraumatic growth in adult patients undergoing treatment for acute leukemia. $\mathrm{J}$ Clin Psychol Med Settings. 2013;20(1):13-24.

38. Lindstrom CM, Cann A, Calhoun LG, Tedeschi RG. The relationship of core belief challenge, rumination, disclosure, and sociocultural elements to posttraumatic growth. Psychol Trauma Theor Res Pract Policy. 2013;5(1):50-5.

39. Irie W, Shiwaku H, Taku K, Suzuki Y, Inoue Y. Roles of reexamination of core beliefs and rumination in posttraumatic growth among parents of children with cancer: Comparisons with parents of children with chronic disease. Cancer Nurs. 2021;44(1):20-8.

40. Butler LD, Blasey CM, Garlan RW, McCaslin SE, Azarow J, Chen XH, et al. Posttraumatic growth following the terrorist attacks of September 11, 2001: Cognitive, coping, and trauma symptom predictors in an internet convenience sample. Traumatology. 2005;11(4):247-67.

41. Zebrack B, Kwak M, Salsman J, Cousino M, Meeske K, Aguilar C, et al. The relationship between posttraumatic stress and posttraumatic growth among adolescent and young adult (AYA) cancer patients. Psychooncology. 2015;24(2):162-8.

\section{Figures}




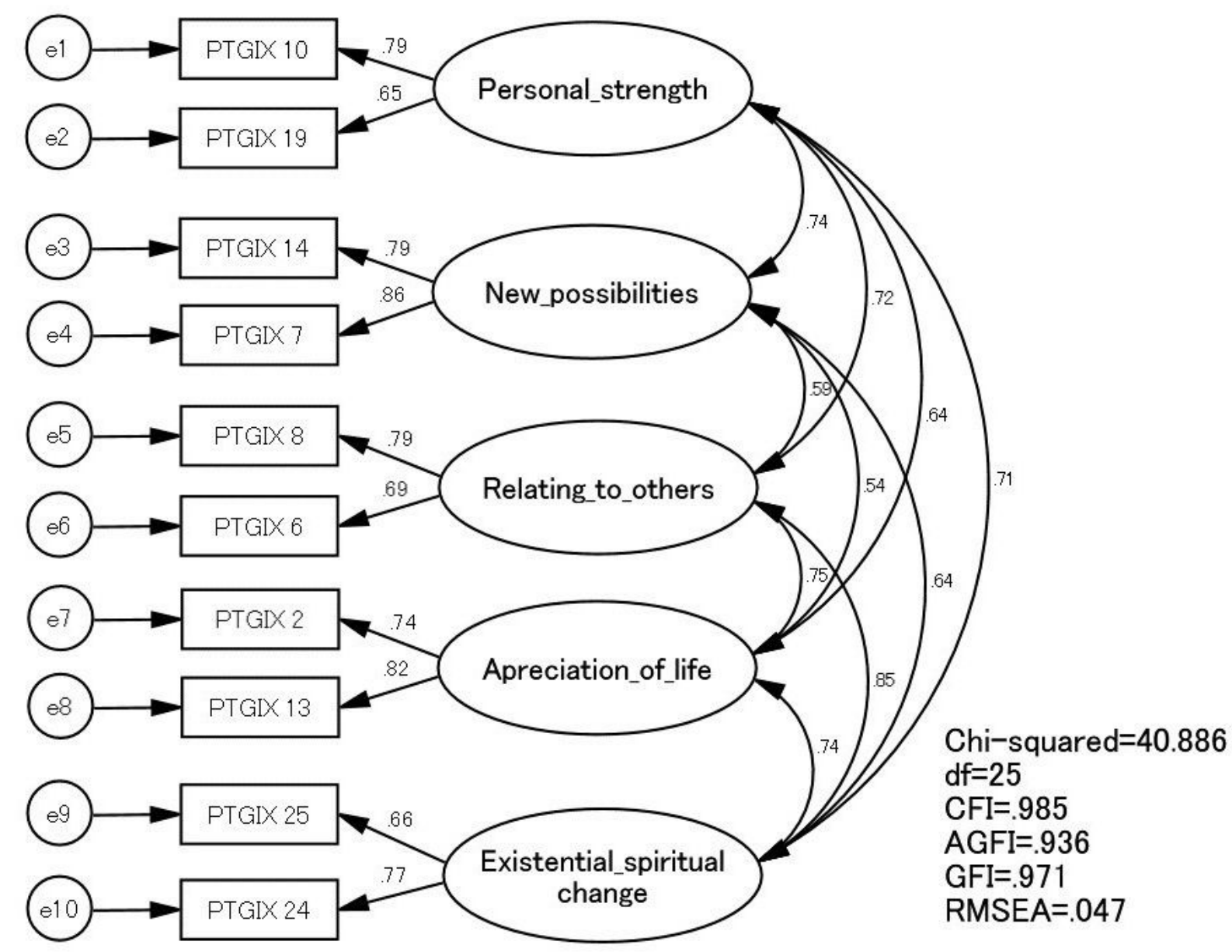

Figure 1

Confirmatory factor analysis using the second sample (284 Japanese students). 